\title{
cop1: a regulatory locus involved in light-controlled development and gene expression in Arabidopsis
}

\author{
Xing-Wang Deng, Timothy Caspar, and Peter H. Quail \\ University of California, Berkeley/U.S. Department of Agriculture Plant Gene Expression Center, Albany, California 94710 \\ USA; Department of Plant Biology, University of California, Berkeley, California 94720 USA
}

\begin{abstract}
Light signals from the environment are perceived by specific regulatory photoreceptors in plants and are transduced by unknown mechanisms to genes that control growth and development. We have identified a genetic locus in Arabidopsis thaliana, which appears to play a central role in this transduction process. Mutations in this locus, designated cop1 (constitutively photomorphogenic), result in dark-grown seedlings with the morphology of wild-type seedlings grown in the light. In addition, these mutations lead to constitutive expression of an array of normally light-regulated genes in dark-grown seedlings and in light-grown adult plants placed in darkness. Promoter-reporter fusion constructs of some of these genes are constitutively expressed in dark-grown transgenic copl seedlings, indicating that the aberrant behavior of these genes results primarily from aberrant modulation of their promoter activities in the mutant. In contrast, light control of seed germination and diurnal control of cab gene expression is normal in the cop 1 mutants. Because these mutations are recessive, we conclude that in seedlings and adult plants, the wild-type cop1 gene product normally acts in darkness to repress the expression of genes involved in the photomorphogenic developmental pathway and to promote the expression of genes involved in the dark-adaptive developmental pathway, and that regulatory photoreceptors act to reverse this action upon exposure to light. However, photocontrol of seed germination and diurnal rhythms is apparently exerted via one or more separate pathways not involving the copl product.
\end{abstract}

[Key Words: Arabidopsis; gene expression; light regulation; photomorphogenesis]

Received March 8, 1991; revised version accepted April 30, 1991.

Light is arguably the single most important environmental factor influencing plant growth and development. Plants not only use incident radiation as an energy source but also as an informational signal to control development in a process termed photomorphogenesis (for review, see Kendrick and Kronenberg 1986). At least three photoreceptors, phytochrome, a blue/UV-A light receptor (also called cryptochrome), and a UV-B light receptor, are utilized to mediate such light-regulated events. Many plant responses mediated by the different photoreceptors are well characterized; and, in some cases, the response has been shown to involve regulation of transcription of specific genes (Tobin and Silverthorne 1985; Kuhlemeier et al. 1987; Kendrick and Kronenberg 1988; Gilmartin et al. 1990). Some genes, many of which are involved in photosynthesis, are induced to high levels upon exposure to light, whereas others, such as those encoding type-A phytochrome, are repressed upon exposure to light (Lissemore and Quail 1988; Sharrock and Quail 1989|. In some cases, the promoter elements involved in light regulation have been delineated /Giuliano et al. 1988b; Manzara and Gruissem 1988; Bruce et al. 1989; Bruce and Quail 1990; Donald and Cashmore
1990; Gilmartin et al. 1990), and protein factors that interact with these elements have been characterized and their mRNAs cloned (Dehesh et al. 1990; Gilmartin et al. 1990). However, little is known about what comprises photoreceptor signal transduction pathways and how they control plant growth and developmental responses through regulated gene expression (Kendrick and Kronenberg 1986; Gilmartin et al. 1990).

Seedling development in Arabidopsis thaliana provides a useful model system for the study of photomorphogenesis of higher plants. First, this plant has welldocumented advantages for genetic and molecular manipulations (for review, see Meyerowitz 1989). In addition, Arabidopsis seedlings, like other higher plants, are genetically endowed with the ability to follow two different strategies of development, skotomorphogenesis and photomorphogenesis, depending on the ambient light conditions (Mohr and Shropshire 1983). Skotomorphogenesis is the developmental strategy followed in darkness. Similar to other dicotyledonous plants, darkgrown Arabidopsis seedlings have long hypocotyls, unopened apical hooks, and undeveloped /small and unopened) cotyledons, with etioplasts and retarded cell- 
type differentiation (e.g., no stomata). In contrast, photomorphogenesis is the developmental strategy followed in the light. Light-grown Arabidopsis seedlings have short hypocotyls, no apical hooks, and open and enlarged cotyledons, with developed chloroplasts and differentiated cell types. There is also a dramatic change in the pattern of gene expression. The light signals perceived by all three known photoreceptor systems apparently contribute to the decision to use either the skotomorphogenic or photomorphogenic pathway (Mohr and Shropshire 1983; Kendrick and Kronenberg 1986).

Two different genetic screening procedures have been employed to identify genes involved in Arabidopsis photomorphogenesis. One approach has been to isolate mutants that show dark-grown morphology when germinated in the light (Koornneef et al. 1980; Adamse et al. 1989; Chory et al. 1989b). Six loci which, when mutated, result in long hypocotyls in the light (hy mutations), have been identified. Three of these loci (hyl, hy2, hy6) result in a deficiency of functional phytochrome (Parks et al. 1989; Chory et al. 1989b). The other approach has been to isolate mutants that show light-grown morphology when germinated in the dark (Chory et al. 1989a). Mutations in the det 1 locus result in a variety of light-grown characteristics in dark-germinated seedlings, including the accumulation of mRNAs for genes that are normally only induced by light. However, mutations in the det locus have little effect on the response of light-regulated genes to dark adaptation of light-grown adult plants (Chory et al. 1989a). This raises the question of whether different sets of genes are involved in the control of different lightregulated developmental processes.

To address the molecular mechanisms underlying signal transduction in Arabidopsis photomorphogenesis, we have chosen to identify genes whose products may play key regulatory roles in this process. We have initiated systematic genetic screening to isolate mutants that show constitutive photomorphogenic (cop) phenotypes, that is, that develop in darkness as wild-type seedlings do in the light. Although the recently identified $\operatorname{det} 1$ mutants also fit these criteria (Chory et al. 1989b), we reasoned that multiple genetic loci are likely to be involved in the complex regulatory circuitry that controls photomorphogenesis and that a full understanding of this circuitry will require characterization of these multiple components. Furthermore, we are also interested in determining whether genes are involved in the control of photomorphogenesis in both seedlings and adult plants or whether separate sets of genes are involved at different developmental stages. Here, we report the identification and characterization of a gene (copl) that appears to play a regulatory role both in the photomorphogenesis of seedlings and in the dark-adaptation response of adult Arabidopsis plants. We have analyzed the characteristics of the copl mutants at the morphologic, cellular, subcellular, and gene expression levels. The question of whether the effects of cop 1 mutations on gene expression are exerted via the promoters of light-responsive genes has been addressed using promoter-reporter gene fusions in transgenic copl plants.

\section{Results}

Isolation of mutants that show constitutive photomorphogenic phenotypes

We have isolated four independent Arabidopsis lines carrying recessive mutant alleles at a locus, designated copl. Details of the mutant isolation procedures and identification of these multiple alleles (cop1-1 through cop 1-4) will be presented elsewhere (X.-W. Deng and P.H. Quail, in prep.). Seedlings with mutations in the cop 1 locus develop in darkness as wild-type seedlings do in the light (Fig. 1). This behavior is similar to that reported previously for the detl and det2 mutants of Arabidopsis (Chory et al. 1989a). Dark-grown cop1 seedlings exhibit short hypocotyls and open and enlarged cotyledons, and accumulate high levels of anthocyanin. However, they lack chlorophyll, because chlorophyll biosynthesis requires light. Mutations in the cop1 locus also have an effect on light-grown plants, resulting in severe dwarfism and low fertility (X.-W. Deng and P.H. Quail, in prep.). Complementation tests of cop 1 with $\operatorname{det} 1$ and det 2 mutants (Table 1) indicate that the cop 1 mutation defines a genetic locus that is different from $\operatorname{det} 1$ and det2. Because the cop 1-1 allele is the first one isolated and the most advanced in backcrossing, it has been used for most of the characterizations reported here.

\section{Dark-grown cop1 seedlings exhibit light-grown characteristics at cellular and subcellular levels}

The constitutive photomorphogenic characteristics of the dark-grown cop 1 mutant are also evident at the cellular level. Unlike dark-grown, wild-type seedlings that have very elongated hypocotyl cells, the hypocotyl cells of dark-grown copl seedlings are short and are even slightly shorter than those of light-grown, wild-type seedlings (data not shown). Cross sections of cotyledons show that the morphologies of dark- and light-grown (Fig. 2A and B, respectively) copl cotyledons are almost identical to each other and very similar to light-grown, wild-type seedlings (Fig. 2D), except that the mutant has less air space between the mesophyll cells. The cotyledons of dark-grown cop 1 seedlings clearly develop stomatal structures in the epidermis and various other differentiated cell types within the organ (Fig. 2A), whereas dark-grown, wild-type seedlings lack both features (Fig. 2C). The cellular basis for cotyledon enlargement in dark-grown copl seedlings as compared with that of wild type is due to both the cell expansion and cell division (Fig. 2A and $\mathrm{C}$.

Plastid morphology in dark- and light-grown copl seedlings was examined by electron microscopy (Fig. 3). Typical plastids in cotyledons of dark- and light-grown wild-type seedlings are shown in Figure 3, A and B, respectively. The morphologies of these plastids are similar to those of other plant species (Kirk and Tilney-Bassett 1978). Plastids from dark-grown seedlings (etioplasts) contain a central paracrystalline assembly of tubules, termed the prolamellar body. Plastids from light-grown seedlings (chloroplasts) have typical thyla- 
A

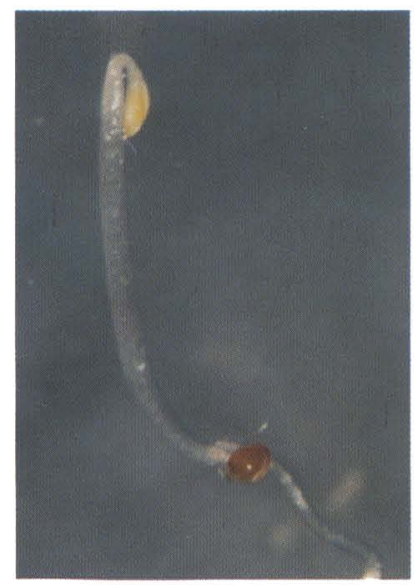

E

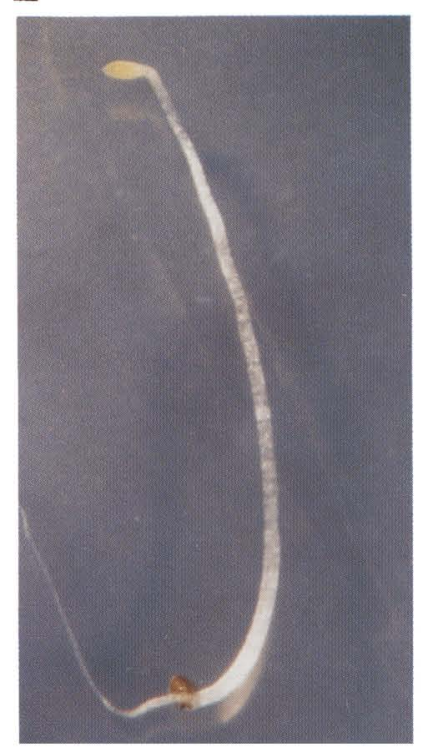

B

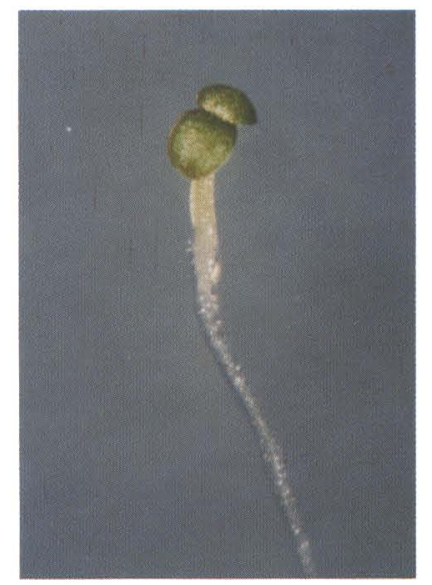

F

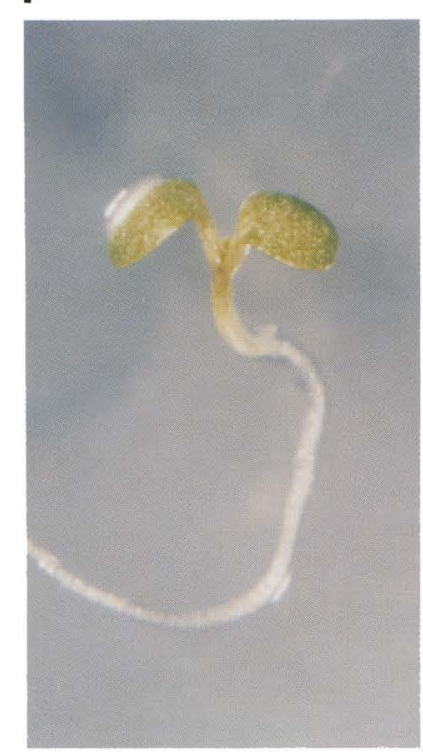

C

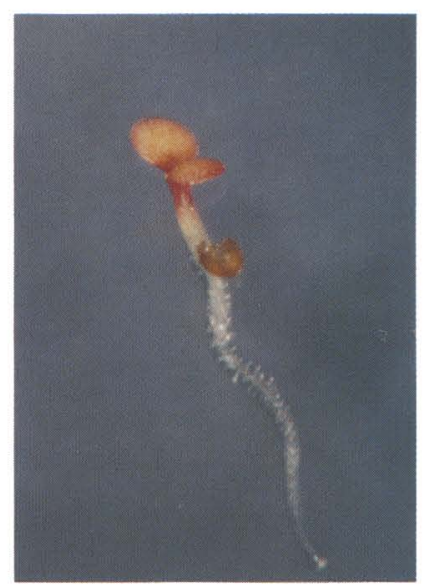

G

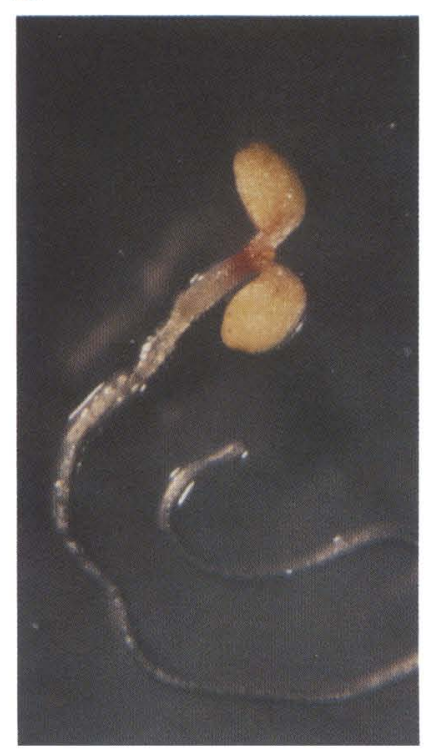

D

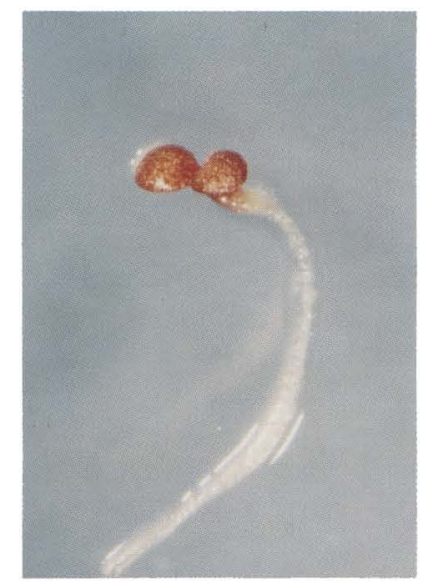

H

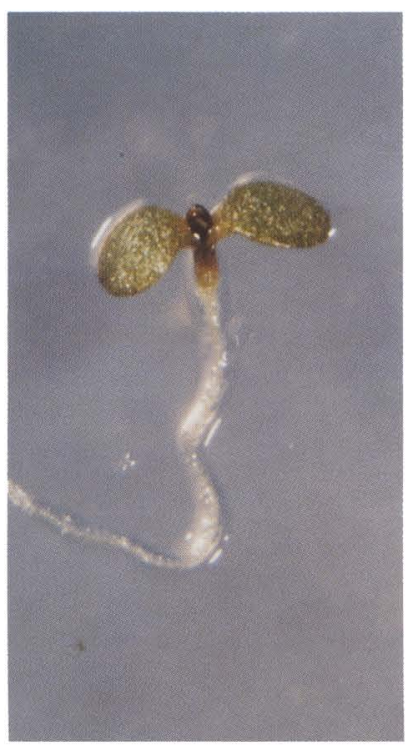

Figure 1. Phenotypes of dark- and light-grown copl and wild-type Arabidopsis seedlings. Seedlings were grown for $3(A-D)$ or $6(E-H)$ days from germination. $(A$ and $E)$ Dark-grown, wild-type seedlings; $(B$ and $F)$ light-grown, wild-type seedlings; $(C$ and $G)$ dark-grown copl-1 seedlings; $(D$ and $H)$ light-grown copl-1 seedlings. The same magnification was used for all panels except $E$, which has two-thirds of the magnification used for the others.

koid membrane structure, composed of stacked and unstacked regions. In Figure 3, C and D, two representative plastids from dark-grown copl-1 and copl-2 seedlings are shown. In contrast with etioplasts of dark-grown, wildtype seedlings, the plastids from dark-grown copl seedlings clearly show signs of chloroplast development, as evidenced by the lack of prolamellar bodies and the formation of some layer two-stacked thylakoid membrane structures. Plastid morphology in cotyledons of lightgrown copl seedlings is identical to that of light-grown, wild-type seedlings (data not shown).

Mutations in the copl locus lead to constitutive expression of light-regulated genes in seedlings and mature plants

The observation that dark-grown copl seedlings appear to have many, if not all, of the physiologic and morphologic characteristics associated with light-grown, wildtype seedlings prompted us to examine whether the expression of light-regulated genes is also altered. We examined, by Northern blot analysis, mRNA levels of eight representative nuclear- and plastid-encoded genes whose expression normally is subjected to light control in both seedlings and adult plants (Fig. 4). Equal amounts of $18 \mathrm{~S}$ cytoplasmic rRNA detected in the different lanes (Fig. 4J) confirms that the same amount of total RNA was used for each sample. The changes in mRNA levels observed in wild-type plants can be divided into three groups. mRNA levels for the five nuclear-encoded, light-inducible genes $(r b c \mathrm{~S}, c a b, f e d \mathrm{~A}, c h s, n i a 2)$ were very low but detectable in dark-grown seedlings, increased substantially in light-grown seedlings and adult plants, and re- 
Table 1. Phenotypic segregation in the $F_{1}$ progeny of crosses between cop1 and det mutations

\begin{tabular}{|c|c|c|c|}
\hline \multirow[b]{2}{*}{$\begin{array}{l}\text { Parental } \\
\text { genotype }\end{array}$} & \multirow[b]{2}{*}{$\begin{array}{c}\text { Germination } \\
\text { rate }(\%)\end{array}$} & \multicolumn{2}{|c|}{$\begin{array}{l}\text { Number of } \\
\text { seedlings }\end{array}$} \\
\hline & & $\begin{array}{l}\text { wild } \\
\text { type }\end{array}$ & mutant \\
\hline $\operatorname{det} 1 / \operatorname{det} 1$ & & & \\
\hline $\begin{array}{l}\quad \times \\
\operatorname{cop} 1-1 / \operatorname{cop} 1-1 \\
\operatorname{det} 2 / \operatorname{det} 2\end{array}$ & 96 & 23 & 0 \\
\hline$\stackrel{\times}{\text { cop } 1-1 / \operatorname{cop} 1-1}$ & 82 & 24 & 0 \\
\hline
\end{tabular}

Seedling phenotypes were examined after growth for 6 days in the dark.

turned to low levels after dark adaptation (Fig. 4A-E). These results are consistent with previous observations for these genes (Chory et al. 1989a; T. Caspar et al., in prep.). Conversely, phyA mRNA levels were high in dark-grown seedlings, decreased in light-grown seedlings and adult plants as reported (Sharrock and Quail 1989), and returned to high levels again after dark adaptation (Fig. 4F). The two plastid-encoded genes $(r b c \mathrm{~L}$ and $p s b \mathrm{~A})$ form their own group (Fig. 4G and H). Like nuclear-encoded, light-inducible genes, the mRNA levels for $r b c \mathrm{~L}$ and $p s b \mathrm{~A}$ were low in dark-grown seedlings and high in light-grown seedlings and adult plants. However, these mRNA levels decreased relatively little, if any, in darkadapted adult plants (two- to threefold for $r b c \mathrm{~L}$ mRNA). Hybridization signals for plastid $16 \mathrm{~S}$ rRNA indicate that these observed differences are not primarily due to major differences in the contribution of total plastid RNA to the total RNA sample (Fig. 4I).

Comparison of the mRNA levels for the light-regulated genes at the same developmental stages in copl and wild-type plants shows that expression in the copl mutant is more nearly constitutive. phyA gene expression is constitutively repressed in dark-grown copl seedlings and in dark-adapted adult cop 1 plants, as it is in wildtype plants in the light (Fig. 4F). Conversely, the mRNA levels of light-inducible nuclear genes are already high in dark-grown seedlings, and, with the exception of chs, do not decrease upon dark adaptation in adult copl plants (Fig. 4A-E). Although the level of chs mRNA in darkadapted cop 1 adult plants is lower than before dark adaptation, it is close to that of light-grown, wild-type seedlings and adult plants (Fig. 4D). In contrast, chs mRNA levels in dark-adapted adult wild-type plants are undetectable, even after longer exposures of Northern blots, under which conditions the chs mRNA in darkgrown, wild-type seedlings is clearly detectable (data not shown). mRNA levels of the two plastid-encoded genes, $r b c \mathrm{~L}$ and $p s b \mathrm{~A}$, in dark-grown cop 1 seedlings are almost as high as those of light-grown seedlings and adult cop 1 plants (Fig. 4G and $\mathrm{H}$ ). These increases in plastid gene mRNA levels in dark-grown cop 1 seedlings are primarily due to gene-specific modulation, but with some possible contribution from the apparent $50 \%$ increase in total plastid RNA level (Fig. 4I).
It is not clear why the absolute levels of $c a b$ and $f e d A$ mRNAs are consistently lower in adult copl plants than those of wild-type adult plants (Fig. 4B and C). On the other hand, high chs mRNA levels in both dark- and light-grown copl seedlings (Fig. 4D) correlate well with the high levels of anthocyanin in these seedlings (see Fig. 1). The low level of anthocyanin in light-grown adult copl plants (data not shown) as compared with copl seedlings also correlates well with chs mRNA levels (Fig. $4 \mathrm{D})$.

\section{Mutations in the cop1 gene activate light-inducible promoters in the absence of light}

The constitutive accumulation of mRNA for light-regu-

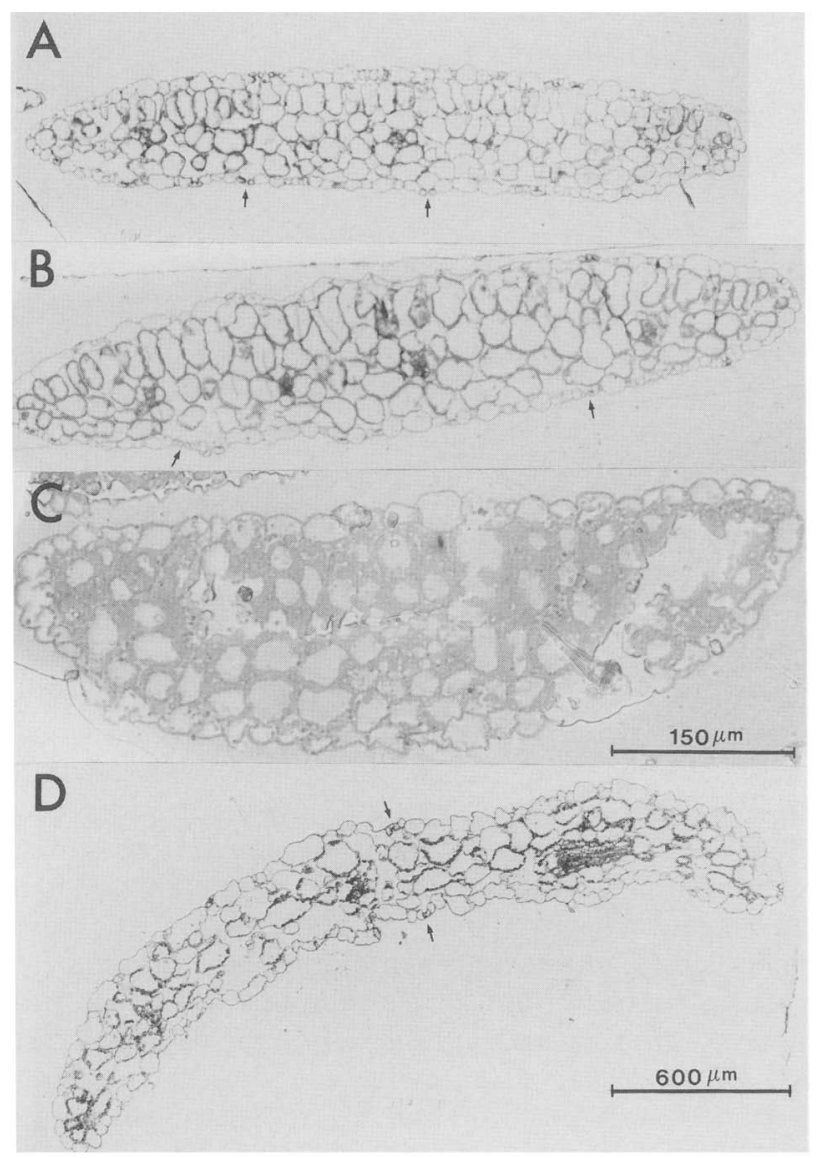

Figure 2. Morphogenetic comparison of cotyledon cross sections from 6-day-old cop1-1 seedlings grown in the dark $\langle A\rangle$ or light $(B)$ and 6-day-old wild-type seedlings grown in the dark $|C|$ or light $(D)$. Light micrographs were taken of $1-\mu \mathrm{m}$ sections of the fixed and embedded seedlings (see Materials and methods). The same magnification was used for all panels (scale shown in $D$ ), except $C$, which was magnified fourfold greater than the others. Examples of stomata are indicated by arrows in $A, B$, and $D$. Note that there are at least twice as many cells per cotyledon in dark-grown copl seedlings $(A)$ as in dark-grown, wild-type seedlings $(C)$ when cross sections from similar positions are compared. 
Figure 3. Morphogenetic changes in plastids of dark-grown seedlings caused by cop 1 mutations. Plastids in cotyledons of 6-day-old dark-grown $(A)$ and light-grown $(B)$ wild-type seedlings, or of 6-day-old cop 1-1 $(C)$ and copl-2 $(D)$ dark-grown mutant seedlings are shown. Typical etioplasts $(A)$ and chloroplasts $(B)$ are present in dark- and light-grown, wild-type seedlings, respectively, whereas the plastids in cotyledons of dark-grown mutants $\mid C$ and $D)$ show similarity to chloroplasts. The magnification scale is indicated for each panel.

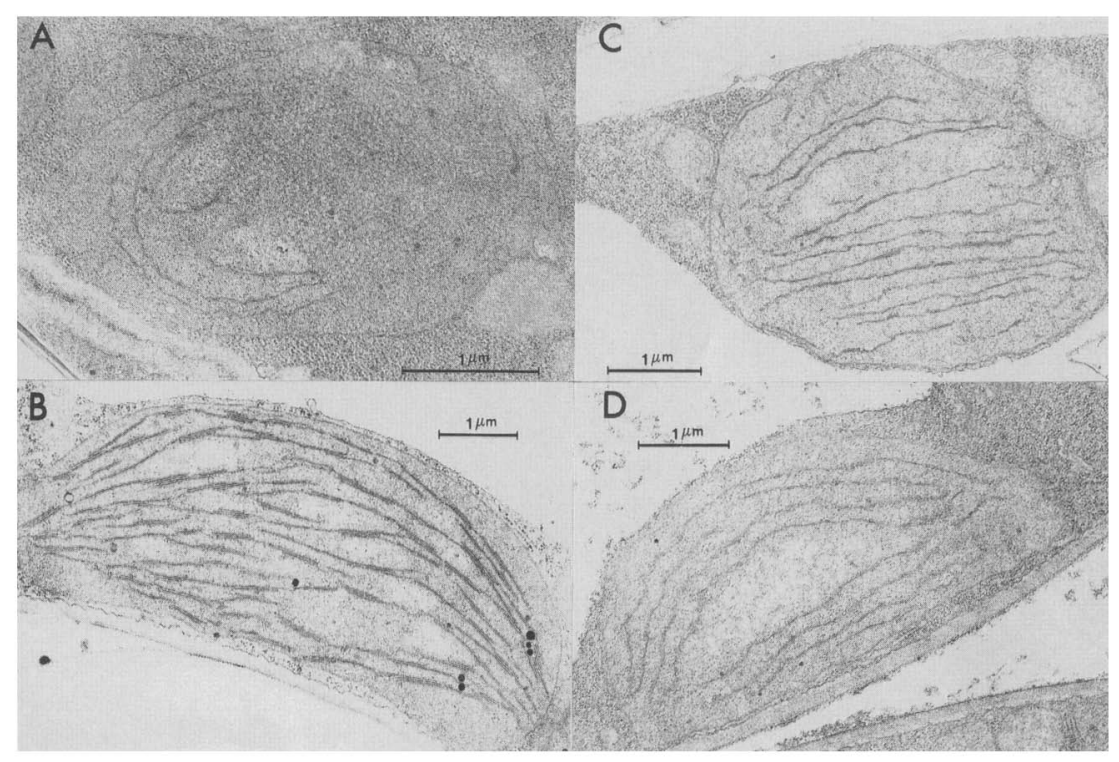

lated genes in dark-grown copl seedlings could result from transcriptional activation of the promoters of these genes. To test this possibility, promoter- $\beta$-glucoronidase (GUS) reporter fusions were constructed (Fig. 5 ; T. Caspar et al., in prep.), transformed into wild-type plants, and introduced into cop 1 mutant plants by conventional genetic crossing (see Materials and methods). At least five transgenic lines, whose GUS expression patterns paralleled those of the endogenous genes, were generated for each construct.

Qualitatively similar results were obtained from different transgenic lines that contained the same promoter-GUS construct. The results obtained from a single representative transgenic line of each construct for both wild-type and mutant plants (see Materials and methods) are shown in Figure 5. The control $35 \mathrm{~S}$ promoter shows slightly higher activities in dark-grown seedlings than in light-grown seedlings in both wild type and mutant (Fig. 5A). However, this difference is considerably less than for the other promoters. The transcriptional activities of both $c a b l$ and fedA full-length promoters were light inducible in wild-type plants (Fig. 5B and $C_{;}$T. Caspar et al., in prep.). The $c a b 1$ promoter activity was induced 20 -fold by light /compared with $>100$-fold induction at the mRNA level; Fig. 4B), whereas the fedA promoter was consistently induced 2to 5 -fold by light (compared with 8 -fold induction at the mRNA level, Fig. 4C; T. Caspar et al., in prep.). The fedA promoter activity in dark-grown copl seedlings is higher than that of light-grown copl seedlings (Fig. 5B). This result indicates that the fedA promoter is fully active in dark-grown mutant seedlings, which is consistent with the result for mRNA levels (Fig. 4C). Qualitatively, $c a b 1$ promoter activity also correlates with mRNA accumulation (Fig. 4B). That is, cabl promoter activity in darkgrown cop1 seedlings is higher than that in dark-grown, wild-type seedlings and lower than that in light-grown $c o p 1$ seedlings. Also similar to the $c a b$ mRNA level changes (Fig.4B), the cabl promoter activity in lightgrown copl seedlings is lower than that of light-grown, wild-type seedlings (Fig. 5C). However, it is difficult to quantitatively correlate the extent of GUS activity induced by light in transgenic plants with endogenous mRNA level changes, because GUS expression may be affected by the chromosomal location of the introduced DNA, the background level of promoter activity under noninductive conditions, and the long half-life of the GUS protein. Because the $c a b$ mRNA level shown in Figure 4 is the sum of three $c a b$ genes (cab1-cab3), it is also possible that the total $c a b$ mRNA level may not necessarily reflect the changes of $c a b l$ mRNA (KarlinNeumann et al. 1988). However, quantification of individual $c a b$ mRNAs in cop 1 plants by $\mathrm{S} 1$ protection analysis indicates that all three mRNAs are likely to be affected by the cop 1 mutation to the same degree /data not shown).

To begin to identify sequences in light-regulated promoters potentially involved in dark activation in the cop 1 mutant, a cabl promoter deletion mutant was fused to GUS and introduced into wild-type and mutant plants (Fig. 5D). This promoter fragment (from -200 to +67 ) has been shown in transgenic tobacco plants to be the minimum sequence required for full light inducibility and tissue-specific expression (Ha and An 1988). Our results confirm that this fragment does confer correct light inducibility ( $>10$-fold induction) and tissue specificity (data not shown) in wild-type Arabidopsis plants. However, the absolute activity of this construct was much reduced in both dark- and light-grown seedlings compared with the full-length promoter. Nevertheless, the activity of this fragment is modulated in copl seedlings in a manner parallel with that of the full-length 


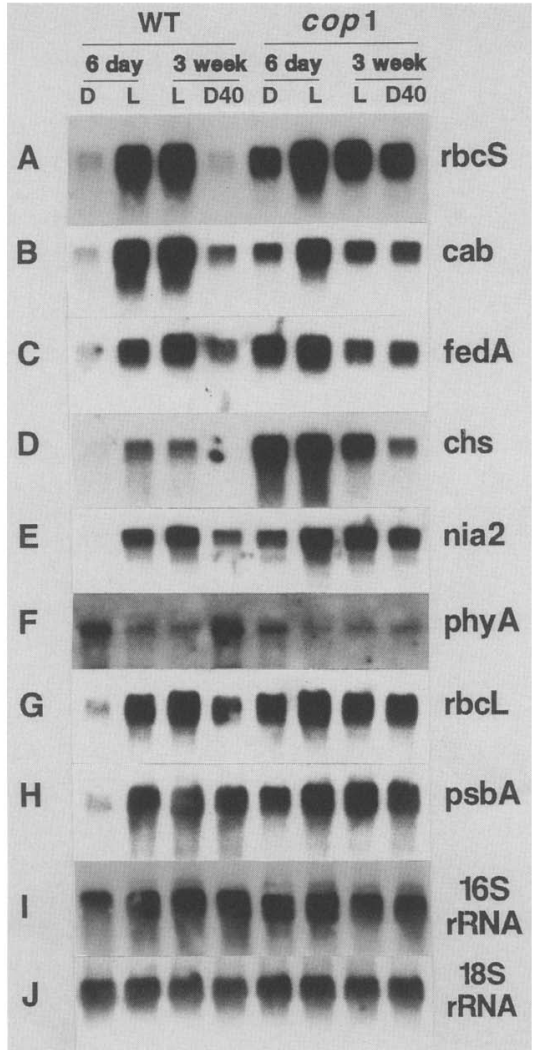

Figure 4. Northern blot analysis of steady-state RNA levels of nuclear- and plastid-encoded genes. RNA levels from wild-type (WT) and cop 1-1 mutant plants were analyzed. Total RNA was isolated from seedlings grown for 6 days in the dark $(D)$ or light (L); from adult plants grown for 3 weeks in continuous light $(\mathrm{L})$; and from adult plants grown for 3 weeks in continuous light and then returned to darkness for $40 \mathrm{hr}$ before harvest (D40). RNA isolation, hybridization probe preparation, and Northern hybridization are described in Materials and methods. Equal amounts of total RNA from the different plant samples were used, and 10 identical blots were hybridized with labeled genespecific probes for 10 different genes: $(r b c S)$ Gene insert for small subunit (SSU) of ribulose-1,5-bisphosphate carboxylase (Krebbers et al. 1990); (cab) gene for chlorophyll a- and b-binding proteins of photosynthetic light-harvesting complexes (Leutwiler et al. 1986); (fedA) ferredoxin type A gene (Somers et al. 1990); (chs) chalcone synthase gene (Feinbaum and Ausubel 1988); (nia2) nitrate reductase gene 2 (Crawford et al. 1988); (phyA) type-A phytochrome gene (Sharrock and Quail 1989); (rbcL) plastid gene encoding the large subunit (LSU) of ribulose-1,5-bisphosphate carboxylase (Zarawski et al. 1981); ( $p s b \mathrm{~A}$ ) plastid gene encoding the $32-\mathrm{kD}$ protein of photosystem II (Zarawski et al. 1982); and genes for plastid (16S rRNA) (Deng and Gruissem 1987) and cytoplasmic (18S rRNA) small rRNAs (Jorgensen et al. 1987). Northern blots were exposed to $\mathrm{X}$-ray film for different periods of time to obtain suitable exposures for each transcript.

promoter (Fig. 5D). These results indicate that this mutant promoter contains sufficient sequence for the dark activation of the $c a b 1$ promoter in copl mutant plants.

The cop1 mutant does not affect phytochrome control of seed germination

The initial germination process /as distinct from subse- quent seedling growth and development) is clearly under phytochrome control in wild-type Arabidopsis seeds (Chory et al. 1989a; X.-W. Deng et al., unpubl.). We therefore examined whether the copl mutation affects phytochrome control of seed germination in addition to seedling development. Seeds from homozygous copl plants were pretreated with different light regimes before germination in darkness (Table 2). Germination rates are high in the green light, red light, and far-red followed by red light treatments. In contrast, germination rates after far-red alone, and red followed by far-red light treatments show a decrease of at least 10 -fold relative to the abovementioned treatments. These results are very similar to those for wild-type seeds (data not shown; Chory et al. 1989a). The inhibition of germination by far-red light and the reversibility of red and far-red treatments unambiguously show that the cop 1 mutation does not affect phytochrome control of seed germination. The high germination rates of unirradiated seeds or seeds treated with green safelight and the reduction in this rate by far-red light alone implies that the amount of active phytochrome in the untreated seeds was sufficient to promote germination without further treatment. These results indicate that separable mechanisms are responsible for induction of seed germination and photomorphogenesis of seedlings, although light and phytochrome are involved in both processes.

\section{The cop1 mutation does not change diurnal fluctuations in cab mRNA levels}

It is well documented that the transcriptional activity of several plant genes is subjected to diurnal control (Giuliano et al. 1988a; Nagy et al. 1988). The best-studied example is the $c a b$ genes. $c a b$ mRNA levels and transcriptional activity fluctuate during the diurnal cycle, reaching the lowest point in the middle of the night and the highest level in early afternoon /Giuliano et al. 1988a; Nagy et al. 1988). This periodic fluctuation is apparently controlled by an endogenous circadian clock, whose phase can be entrained by light, possibly through the phytochrome system /Giuliano et al. 1988a; Nagy et al. 1988; Lam and Chua 1989). We examined whether the cop 1 mutation affects diurnal control of $c a b$ mRNA levels. Figure 6 shows that $c a b$ mRNA levels in the copl mutant fluctuate with the same periodicity as those in wild-type plants, albeit with a lower amplitude. These results indicate that the wild-type copl gene product appears to be involved only in light regulation of $c a b$ gene expression and not in diurnal control of its expression. It should be noted that the adult wild-type and copl plants used for the dark-adaptation experiments shown in Figure 4 were grown under continuous light ( $24 \mathrm{hr} /$ day) to suppress the diurnal fluctuations in mRNA levels for simplification of the dark-adaptation studies.

\section{Discussion}

Here, we report the characterization of a genetic locus, designated cop 1 , which plays a regulatory role in the 
Figure 5. Modulation of promoter activity by light and by the copl mutation. The promoter-GUS fusion constructs used for genetic transformations are shown at left, and the GUS activities (nmoles 4-methylumbelliferone/hr per $\mathrm{mg}$ of protein) of transgenic wild-type (wt) or mutant seedlings containing these constructs grown either in dark or light are shown at right. The four promoters used are a 346-bp CaMV 35S promoter fragment (Rogers et al. 1986) (A), a 1.9-kb promoter fragment from the Arabidopsis fedA gene (Sommers et al. 1990; T. Caspar et al. in prep. $)(B)$, a 1.35 -kb promoter fragment from the Arabidopsis cabl promoter ( $\mathrm{Ha}$ and An 1988) $|C|$, and a 267-bp minimal promoter fragment from the $c a b l$ gene $(D)$. The details of promoter-GUS fusion construction, genetic transformation, and GUS activity assay are described in Materials and methods. In each promoter-GUS construct, the end positions (base pairs) of the promoter fragment are marked relative to the transcription start site $(+1)$. GUS activities are the average of three independent measurements, among which the variation was $<5 \%$.

control of photomorphogenesis in Arabidopsis, both in seedlings and adult plants. Dark-grown copl seedlings exhibit seedling morphology, cell and organelle differentiation, and gene expression - that are normally observed only in light-grown, wild-type seedlings (Kendrick and Kronenberg 1986; Kuhlemeier et al. 1987). The recessive nature of the four mutant alleles obtained implies that a loss of function leads to the constitutive photomorphogenic phenotype. These observations suggest that the wild-type copl gene is an early regulatory gene in the cascade of changes that control photomorphogenesis in Arabidopsis. The encoded product (COP1) of the wildtype cop 1 locus appears to have a critical role in coupling light perception to morphogenesis and, therefore, in switching between skotomorphogenic and photomorphogenic pathways during seedling development. On the other hand, the severe defects observed in adult lightgrown copl plants (X.-W. Deng and P.H. Quail, in prep.) suggest that the cop 1 locus also plays a role in the development of light-grown plants.

The morphology of dark-grown copl seedlings is similar to that reported recently for detl mutants, which also have a light-grown phenotype when grown in the dark (Chory et al. 1989a). However, the two mutations are at different genetic loci (Table 1) on two different

Table 2. Germination rates for cop1 mutant seed under different light conditions

\begin{tabular}{lccc}
\hline Light treatment & Seeds & Seedlings & $\begin{array}{c}\text { Germination } \\
\text { rate }(\%)\end{array}$ \\
\hline Dark & 123 & 66 & 54 \\
Green safelight & 111 & 59 & 53 \\
Red & 125 & 84 & 67 \\
Far-red & 115 & 6 & 5 \\
Red/far-red & 100 & 5 & 5 \\
Far-red/red & 112 & 63 & 56 \\
\hline
\end{tabular}

The light treatments were green safelight, 2 min; red, $5 \mathrm{~s}$ saturating pulse; far-red, $10 \mathrm{~s}$ saturating pulse. In the case of two different light treatments, the second light exposure immediately followed the first exposure. chromosomes (X.-W. Deng and P.H. Quail, in prep.) and there are two distinct regulatory differences between these two classes of mutants. First, copl does not affect normal phytochrome control of seed germination (Table 2 ), whereas $\operatorname{det} 1$ seeds germinate regardless of the presence or absence of active phytochrome (Chory et al. 1989a). Second, adult cop 1 mutant plants do not display a decrease or increase in the mRNA levels of light-regulated genes upon dark adaptation (Fig. 4) in contrast with wild-type and det 1 plants (Chory et al. 1989a). The det 1 gene product is apparently involved in light regulation of germination and seedling development but not in dark adaptation, whereas the copl product is involved in light regulation in seedling development and dark adaptation of adult plants, but not in seed germination. Thus, different regulatory circuitry may mediate the light regulation of seed germination, seedling development, and dark-adaptation processes. On the other hand, a given regulatory component (such as $\operatorname{det} 1$ and cop1) could be

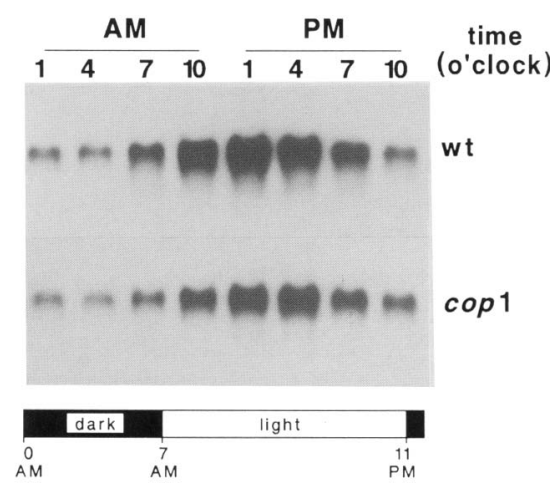

Figure 6. Diurnal fluctuation of $c a b$ mRNA levels in wild-type (wt) and cop 1 mutant plants. Wild-type and cop 1 mutant plants were grown under a $16-\mathrm{hr}$ light/8-hr dark (11 p.m. to 7 a.m.) photoperiod for 21 days. The plants were then harvested at $3-\mathrm{hr}$ intervals for $24 \mathrm{hr}$ as shown. Total RNA was isolated from these plants, and $5 \mu \mathrm{g}$ from each sample was used for Northern analysis of $c a b$ mRNA (see Materials and methods). 
involved in more than one of the regulatory circuitries. It will be interesting to know how det 1 and copl relate to each other and how they regulate photomorphogenesis.

The cop 1 mutation affects plant responses that are mediated by all three photoreceptor systems. These responses include inhibition of hypocotyl elongation (Koornneef et al. 1980; Kendrick and Kronenberg 1986) and modulation of gene expression ( $c a b$, Karlin-Neumann et al. 1988; fedA and nia2, T. Caspar et al., in prep.; chs, Chory et al. 1989a). Therefore, the function of COP1 does not appear to be restricted to the signal transduction pathway of a single photoreceptor, implying that the photoreceptors or their transduction pathways merge at some point to modulate the activity of COP1.

The fact that the germination rate of $\operatorname{cop} 1$ mutant seeds is still fully under phytochrome control (Table 2) suggests that both the relevant phytochrome and the transduction pathway leading to this response are unaffected by the cop 1 mutation. It also suggests that germination and photomorphogenesis during seedling development involve two independent developmental commitments. Although phytochrome modulates both commitments, the data indicate either that there is a branchpoint in the phytochrome signal transduction pathway, after which different components are involved in controlling the two processes, or that two separate types of phytochrome with independent signal transduction pathways operate in parallel to control the two processes (see Smith and Whitelam 1990). Similarly, the normal periodicity in the diurnal fluctuation of $c a b$ mRNA levels in cop 1 mutants suggests that COP1 is not involved in the diurnal control of $c a b$ gene expression.

The expression of a variety of plant nuclear genes is either positively or negatively regulated by the presence of light, the effects of which are mediated by either phytochrome (such as cab, Karlin-Neumann et al. 1988; phyA, Lissemore and Quail 1988; fedA and nia2, T. Caspar et al., in prep.) or the blue-light receptor (such as chs, Chory et al. 1989a). It has been demonstrated for most of these genes that light modulates transcriptional activity through their promoter sequences (Lissemore and Quail 1988; Bruce et al. 1989; Gilmartin et al. 1990; T. Caspar et al., in prep.). On the basis of our analysis of mRNA levels and promoter-GUS constructs in transgenic plants (Figs. 4 and 5), we propose that in wild-type plants, COP1 is involved in the modulation of promoter activity of light-regulated genes. To achieve this, COP1 must, in darkness, concomitantly suppress the transcriptional activity of light-inducible promoters (by activation of a repressor or inhibition of an activator) and activate or derepress the transcriptional activity of light-repressible promoters. Light is thus postulated to repress COP1 function via photoreceptor activation, as do mutations in the copl gene.

Overall, our experimental results and current knowledge of photomorphogenesis (Kendrick and Kronenberg 1986; Adamse et al. 1988; Gilmartin et al. 1990) are consistent with the following concept of light-regulated development in Arabidopsis. Seed germination and seedling development involve two distinct decisions: the first involving germination; and the second involving a choice between two developmental strategies-skotomorphogenesis and photomorphogenesis (Mohr and Shropshire 1983). A set of control genes (such as det 1 and cop1) are involved in making these decisions. Light signals are perceived by regulatory photoreceptors and are transduced by still unknown transduction pathways to modulate the activities of the products of these control genes and thereby affect these developmental decisions. In the case of copl, the fact that the mutations are recessive indicates that loss of COPl function is responsible for the switch from skotomorphogenesis to photomorphogenesis in the mutants in darkness. Perception and transduction of light signals in some way abrogates COP1 activity leading to a phenocopy in wild-type plants of the copl mutation.

\section{Materials and methods}

\section{Plant materials and growth conditions}

A. thaliana ecotype Columbia was the parental strain used for the isolation of photomorphogenic mutants. Wild-type seeds and some M2 seed batches mutagenized with ethylmethanesulfonate (EMS) were purchased from Lehle Seeds (Tucson, AZ). Our own EMS-treated M2 seeds were produced according to Sommerville and Ogren (1982).

Unless specified otherwise, Arabidopsis seeds were routinely surface-sterilized for $\sim 15 \mathrm{~min}$ in $30 \%$ bleach (Clorox), rinsed at least five times, and plated in petri plates $(150 \times 25 \mathrm{~mm})$ containing growth medium (GM; Valvekens et al. 1988). After cold treatment at $4^{\circ} \mathrm{C}$ for $2-4$ days in the dark, the plates were incubated in a growth chamber at $22^{\circ} \mathrm{C}$ in complete darkness or in a cycle of $16 \mathrm{hr}$ light $/ 8 \mathrm{hr}$ darkness. For the dark-adaptation experiment, Arabidopsis plants were grown in continuous light ( $24 \mathrm{hr} /$ day) for 3 weeks. Arabidopsis plants in petri dishes were either harvested for experiments or transferred to soil to grow to maturity for genetic manipulations or seed set. For all reported experiments, except the seed germination study, seeds (mixture of mutant and wild type) from plants heterozygous for copl mutations were used. Wild-type and mutant plants were harvested individually and pooled when necessary to assure the same growth conditions for wild-type and cop 1 mutants. The light source for Arabidopsis plant growth was a combination of fluorescent and incandescent lights, ranging from 100 to 300 $\mu$ Einstein $/ \mathrm{m}^{2}$ per sec. For mutant screening, $\sim 2000$ seeds were placed in each plate and incubated in the dark at $22^{\circ} \mathrm{C}$ for 7 days after cold treatment. To study the effect of light on seed germination, seeds from plants homozygous for the cop 1 mutation were used. Sources of specific wavelengths of lights (red, far-red, green safelight) were identical to those published (Bruce et al. 1989).

\section{Analysis of anthocyanin and chlorophyll pigments}

Anthocyanin content in Arabidopsis seedlings was measured by the procedure of Schmidt and Mohr (1981), using $0.5 \mathrm{ml}$ of extraction media for 20-40 light-grown seedlings or 60-120 dark-grown seedlings. For chlorophyll measurement, 20-60 Arabidopsis seedlings were harvested in a microcentrifuge tube containing $0.5 \mathrm{ml}$ of $80 \%$ acetone. The seedlings were ground with a ground-glass rod until the chlorophyll was completely extracted. The extract was then centrifuged at maximum speed in a microcentrifuge for $10 \mathrm{~min}$, and the supernatant was used to measure chlorophyll a and chlorophyll b (Arnon 1949). 


\section{Light and electron microscopy}

The fixation and embedding of Arabidopsis seedlings was performed according to a published procedure (Deng and Gruissem 1987|. Seedlings grown on petri plates for 6 days in the dark or light were harvested and immediately subjected to fixation. The embedded cotyledons were sectioned using an ultramicrotome (model MT 6000-XL from RMC, Inc.). Thick sections (1 $\mu \mathrm{m}$ ) were stained with toluidine blue for light microscopy examinations. Thin sections $(0.1-0.2 \mu \mathrm{m})$ were poststained with $5 \%$ (wt/vol) uranyl acetate in water for $20 \mathrm{~min}$ and counterstained with $3 \%(\mathrm{wt} / \mathrm{vol})$ lead citrate for $10 \mathrm{~min}$. After the sections were dried, the plastids in these sections were examined and photographed using a transmission electron microscope.

\section{RNA analysis}

For 6-day-old dark- and light-grown Arabidopsis seedlings, wild-type or copl-1 mutant individuals were harvested from petri plates and frozen immediately in liquid nitrogen. For 3week-old mature plants, wild-type and mutant individuals that had been separated onto different petri plates, when 5 days old, were pooled and frozen in liquid nitrogen at the specified time points. Dark-grown and dark-adapted plants were harvested under dim green safelight. Isolation of total RNA, electrophoresis and blotting of RNA, and hybridization with radioactively labeled DNA probes were as described previously (Sharrock and Quail 1989). The DNA probes used were a $0.55 \cdot \mathrm{kb}$ DNA fragment [generated by polymerase chain reaction (PCR)] corresponding to the entire open reading frame of the Arabidopsis small subunit of ribulose-1,5-bisphosphate carboxylase gene (rbcS; Krebbers et al. 1988); a 0.5-kb BamHI-SstI DNA fragment corresponding to the coding region of the Arabidopsis cab3 gene (Leutwiler et al. 1986); a 1.5-kb HincII-EcoRI DNA fragment containing the Arabidopsis fedA gene (Somers et al. 1990); a 0.95-kb cDNA coding for the Arabidopsis chalcone synthase gene (chs, Feinbaum and Ausubel 1988); a 0.9-kb DNA fragment (generated by PCR) encoding the first 300 amino acids of an Arabidopsis nitrate reductase gene (nia2, Crawford et al. 1988), a $0.2-\mathrm{kb}$ DNA fragment derived from the Arabidopsis phytochrome A (phyA) gene 3'-noncoding sequence (Sharrock and Quail 1989); a 1.25-kb PstI-EcoRI DNA fragment containing most of the coding region of a spinach chloroplast $r b c L$ gene (Zurawski et al. 1981); a 1.2. kb BgIII-XbaI DNA fragment containing most of the coding region of a spinach chloroplast $p s b \mathrm{~A}$ gene (Zurawski et al. 1982); a 3.0-kb BamHI DNA fragment from the spinach chloroplast genome containing the entire $16 \mathrm{~S}$ rRNA gene (Deng and Gruissem 1988); and a 1.0-kb EcoRIBamHI DNA fragment containing the coding region of the pea nuclear 18S rRNA gene (Jorgensen et al. 1987). The identity of cloned DNA fragments produced by PCR (rbcS, nia2) was confirmed by sequencing. For all probes except phyA, $\sim 200 \mathrm{ng}$ of each purified DNA fragment was labeled to high specific activity $\left(\sim 2 \times 10^{5}\right.$ to $3 \times 10^{5} \mathrm{cpm} / \mathrm{ng}$ of DNA $)$ by random oligomer priming, denatured by boiling for $5 \mathrm{~min}$, and used for hybridization. For the phyA probe, a single-stranded DNA probe was prepared by using a single-stranded DNA template of an M13mp18 clone shown to contain sequence specific for the phyA gene (Sharrock and Quail 1989).

\section{Promoter-GUS constructs and genetic transformation}

A $2.3-\mathrm{kb}$ fragment containing the GUS-coding sequence and the 3 ' sequence from the nopoline synthase (NOS) gene (Jefferson et al. 1987) was inserted into the filled-in HindIII site of the pcit20 vector (a derivative of pMON754; Rogers et al. 1986; obtained from Dr. E. Meyerowitz, California Institute of Technologyl, to create plasmid CGUSA (T. Caspar et al., in prep.). Promoter DNA fragments were inserted upstream of the GUS-coding sequence of CGUSA (see Fig. 5). These included a fragment from -340 to +6 bp from the 35 S cauliflower mosaic virus (CaMV) promoter (Rogers et al. 1986), a fragment from -1850 to $+77 \mathrm{bp}$ from the Arabidopsis fedA promoter (Somers et al. 1990; T. Caspar et al., in prep.), a fragment from -1281 to $+67 \mathrm{bp}$ from the Arabidopsis cabl gene ( $\mathrm{Ha}$ and An 1988; Karlin-Neumann et al. 1988), and a minimal (from -200 to $+67 \mathrm{bp}$ ) Arabidopsis $c a b l$ promoter. For each promoter, the position of the transcriptional initiation site is designated +1 . These constructs were introduced into Arabidopsis (ecotype No-O) using Agrobacterium-mediated transformation of root explants according to Valvekens et al. (1988). At least five independent transgenic Arabidopsis plants were obtained for each construct. Two representative lines for each promoter-GUS fusion construct that produced the expression pattern expected of the endogenous genes were chosen to cross with copl plants. The $F_{2}$ seeds produced by selfing the $F_{1}$ plants, which consisted of one-quarter mutant and three-quarters wild type, were germinated and grown for 6 days under various light conditions, and 20-100 seedlings (both mutants and wild type) were collected, pooled, and assayed for GUS activity. Because both mutant and wildtype seedling pools were randomly collected from the same F2 population, any variation that might be contributed by the genetic backgrounds of the respective parents should have been minimized. With the exception of one line that was transformed with a 35S-GUS construct, the T-DNA insert sites appeared to be unlinked to the copl locus in the transgenic lines chosen.

\section{GUS activity assay}

Tissue-specific expression of introduced promoter-GUS constructs was monitored by histochemical staining. Briefly, Arabidopsis seedlings were harvested and immersed in $50 \mathrm{~mm}$ phosphate buffer containing $1 \mathrm{mM}$ 5-bromo-4-chloro-3-indolyl- $\beta$-glucuronide (X-Gluc), frozen at $-80^{\circ} \mathrm{C}$, thawed at room temperature, and incubated at $37^{\circ} \mathrm{C}$ for $2-24 \mathrm{hr}$, depending on the amount of GUS activity present in the seedlings. GUS enzyme activity in transgenic Arabidopsis seedlings was quantified according to Jefferson (1987) by measuring the fluorescence of methylumbelliferone ( $\mathrm{MU}$ ) produced by GUS cleavage of methylumbelliferyl- $\beta$-D-glucuronide. Protein concentration was determined by the Bio-Rad protein assay (Bio-Rad) according to the manufacturer's suggested procedure.

\section{Acknowledgments}

We thank Dr. Joanne Chory for exchanging $\operatorname{det} 1$ and $\operatorname{det} 2 \mathrm{mu}$ tants with our copl mutant for genetic complementation tests. We also thank Angela Chu and Ja Moon for their assistance with Arabidopsis planting and harvesting; David Somers for providing the $c a b$ and $c h s$ DNA probes; Margaret Boylan for providing the 35S-GUS construct; Xue-Lan Wang and the staff of electron microscope laboratory at University of California, Berkeley, for their technical assistance with the microscopy analysis; and Drs. Wilhelm Gruissem and Sarah Hake for their critical reading of the manuscript. This work was supported by U.S. Department of Agriculture/ARS CRIS 5335-23000-004-00D (to P.H.Q.) and a postdoctoral fellowship grant from the Life Sciences Research Foundation (to X.-W.D.). X.-W.D. is a DuPont Fellow of the Life Sciences Research Foundation. T.C. is a postdoctoral fellow of the National Science Foundation.

The publication costs of this article were defrayed in part by 
payment of page charges. This article must therefore be hereby marked "advertisement" in accordance with 18 USC section 1734 solely to indicate this fact.

\section{References}

Adamse, P., R.E. Kendrick, and M. Koornneef. 1988. Photomorphogenetic mutants of higher plants. Photochem. Photobiol. 48: $833-841$.

Arnon, D.I. 1949. Copper enzymes in isolated chloroplasts: Polyphenoloxidase in Beta vulgaris. Plant Physiol. 24: 1-13.

Bruce, W.B. and P.H. Quail. 1990. cis-Acting elements involved in photoregulation of an oat phytochrome promoter in rice. Plant Cell 2: 1081-1089.

Bruce, W.B., A. Christensen, T. Klein, M. Fromm, and P.H. Quail. 1989. Photoregulation of a phytochrome gene promoter from oat transferred into rice by particle bombardment. Proc. Natl. Acad. Sci. 86: 9692-9696.

Chory, I., C. Peto, R. Feinbaum, L. Pratt, and F. Ausubel. 1989. Arabidopsis thaliana mutant that develops as a light-grown plant in the absence of light. Cell 58: 991-999.

Chory, J., C.A. Peto, M. Ashbaugh, R. Saganich, L. Pratt, and F. Ausubel. 1989b. Different roles for phytochrome in etiolated and green plants deduced from characterization of Arabidopsis thaliana mutants. Plant Cell 1: 867-880.

Crawford, N.M., M. Smith, D. Bellissimo, and R.W. Davis. 1988. Sequence and nitrate regulation of the Arabidopsis thaliana mRNA encoding nitrate reductase, a metalloflavoprotein with three functional domains. Proc. Natl. Acad. Sci. 85: 5006-5010.

Dehesh, K., W.B. Bruce, and P.H. Quail. 1990. A trans-acting factor that binds to a GT-motif in a phytochrome gene promoter. Science 250: 1397-1399.

Deng, X.-W. and W. Gruissem. 1987. Control of plastid gene expression during development: The limited role of transcriptional regulation. Cell 49: 379-387.

- 1988. Constitutive transcription and regulation of gene expression in non-photosynthetic plastids of higher plants. EMBO I. 7: 3301-3308.

Donald, R.G.K. and A.R. Cashmore. 1990. Mutation of either G box or I box sequences profoundly affects expression from the Arabidopsis rbcS-1A promoter. EMBO J. 9:1717-1726.

Feinbaum, R.L. and F.M. Ausubel. 1988. Transcriptional regulation of the Arabidopsis thaliana chalcone synthase gene. Mol. Cell. Biol. 8: 1985-1992.

Gilmartin, P.M., L. Sarokin, J. Memelink, and N.-H. Chua. 1990. Molecular light switches for plant genes. Plant Cell 2: 369-378.

Giuliano, G., N.E. Hoffman, K. Ko, P.A. Scolnik, and A.R. Cashmore. 1988a. A light-entrained circadian clock controls transcription of several plant genes. EMBO J. 7: 3635-3642.

Giuliano, G., E. Pichersky, V.S. Malik, M.P. Timko, P.A. Scolnik, and A.R. Cashmore. 1988b. An evolutionarily conserved protein binding sequence upstream of a plant light-regulated gene. Proc. Natl. Acad. Sci. 85: 7089-7093.

Ha, S.-B. and G. An. 1988. Identification of upstream regulatory elements involved in the developmental expression of the Arabidopsis thaliana cabl gene. Proc. Natl. Acad. Sci. 85: 8017-8021.

Jefferson, R.A. 1987. Assaying chimeric genes in plants: The Gus gene fusion system. Plant Mol. Biol. Rep. 5: 387-405.

Jefferson, R.A., T.A. Kawvanagh, and M.W. Bevan. 1987a. Gus fusions: Beta-glucoronidase as a sensitive and versatile gene fusion marker in higher plants. EMBO I. 6: 3901-3907.

Jorgensen, R.A., R.E. Cuellar, W.F. Thompson, and T.A. Ka- vanagh. 1987. Structure and variation in ribosomal RNA genes of pea: Characterization of a cloned rDNA repeat and chromosomal rDNA variants. Plant Mol. Biol. 8: 3-12.

Karlin-Neumann, G.A., L. Sun, and E.M. Tobin. 1988. Expression of light-harvesting chlorophyll a/b-protein genes is phytochrome-regulated in etiolated Arabidopsis thaliana seedlings. Plant Physiol. 88: 1323-1331.

Kendrick, R.E. and G.H.M. Kronenberg. 1986. Photomorphogenesis in plants. Martinus Nijhoff/Dr W. Junk Publishers, Dordrecht, The Netherlands.

Kirk, J.T.O. and R.A.E. Tilney-Bassett. 1978. The plastids: Their chemistry, structure, growth, and inheritance. Elsevier/ North-Holland Biomedical Press, New York.

Koornneef, M., E. Rolff, and C.J.P. Spruit. 1980. Genetic control of light-inhibited hypocotyl elongation in Arabidopsis thaliana (L.) Heynh. Z. Pflanzenphysiol. 100: 147-160.

Krebbers, E., J. Seurinck, L. Herdies, A.R. Cashmore, and M.P. Timko. 1988. Four genes in two diverged subfamilies encode the ribulose-1,5-bisphosphate carboxylase small subunit polypeptides of Arabidopsis thaliana. Plant Mol. Biol. 11: 745-759.

Kuhlemeier, C., P.J. Green, and N.-H. Chua. 1987. Regulation of gene expression in higher plants. Annu. Rev. Plant Physiol. 38: $221-257$.

Lam, E. and N.-H. Chua. 1989. Light to dark transition modulates the phase of antenna chlorophyll protein gene expression. I. Biol. Chem. 264: 20175-20176.

Leutwiler, L.S., E.M. Meyerowitz, and E.M. Tobin. 1986. Structure and expression of three light-harvesting chlorophyll a/ b-binding protein genes in Arabidopsis thaliana. Nucleic Acids Res. 14: 4051-4064.

Lissemore, J. and H.P. Quail. 1988. Rapid transcriptional regulation by phytochrome of the genes for phytochrome and chlorophyll a/b-binding protein in Avena sativa. Mol. Cell. Biol. 8: 4840-4850.

Manzara, T. and W. Gruissem. 1988. Organization and expression of the genes encoding ribulose-1,5-bisphosphate carboxylase in higher plants. Photosynth. Res. 16: 117-139.

Meyerowitz, E.M. 1989. Arabidopsis, a useful weed. Cell 56: $263-269$.

Mohr, H. and W. Shropshire, Jr. 1983. An introduction to photomorphogenesis for the general reader. In Encyclopedia of plant physiology, new series, vol. 16A. Photomorphogenesis (ed. W. Shropshire Jr. and H. Mohr), pp. 24-38. SpringerVerlag, Berlin/Heidelberg/New York/Tokyo.

Nagy, F., S.A. Kay, and N.-H. Chua. 1988. A circadian clock regulates transcription of the wheat cab-I gene. Genes \& Dev. 2: 376-382.

Parks, B.M., J. Shanklin, M. Koornneef, R.E. Kendrick, and P.H. Quail. 1989. Immunochemically detectable phtyochrome is present at normal levels but is photochemically nonfunctional in the hyl and hy 2 long hypocotyl mutants of Arabidopsis. Plant Mol. Biol. 12: 425-437.

Rogers, S.G., H.J. Klee, R.B. Horsch, and R.T. Fraley. 1986. Gene transfer in plants: Production of transformed plants using $\mathrm{Ti}$ plasmid vectors. Methods Enzymol. 118: 627-640.

Schmidt, R. and H. Mohr. 1981. Time-dependent changes in the responsiveness to light of phytochrome-mediated anthocyanin synthesis. Plant Cell Environ. 4: 433-437.

Sharrock, R.A. and P.H. Quail. 1989. Novel phytochrome sequences in Arabidopsis thaliana: Structure, evolution, and differential expression of a plant regulatory photoreceptor family. Genes \& Dev. 3: 1745-1757.

Smith, H. and G.C. Whitelam. 1990. Phytochrome, a family of photoreceptors with multiple physiological roles. Plant Cell Environ. 13: 695-707. 
Deng et al.

Somers, D.E., T. Caspar, and P.H. Quail. 1990. Isolation and characterization of a ferredoxin gene from Arabidopsis thaliana. Plant Physiol. 93: 572-577.

Somerville, C.R. and W.L. Ogren. 1982. Isolation of photorespiration mutants in Arabidopsis thaliana. In Methods in chloroplast molecular biology (ed. M. Edelman, R.B. Hallick, and N.-H. Chua, pp. 129-139. Elsevier Biomedical Press, New York.

Tobin, E. and I. Silverthorne. 1985. Light regulation of gene expression in higher plants. Annu. Rev. Plant Physiol. 36: 569-593.

Valvekens, D. M. Van Montagu, and M. Van Lusebettens. 1988. Agrobacterium tumefaciens-mediated transformation of $A r$ abidopsis thaliana root explants by using kanamycin selection. Proc. Natl. Acad. Sci. 85: 5536-5540.

Zurawski, G., B. Perrot, W. Bottomley, and P.R. Whitfeld. 1981. The structure of the gene for the large subunit of ribulose1,5-bisphosphate carboxylase from spinach chloroplast DNA. Nucleic Acids Res. 9: 3251-3270.

Zurawski, G., H. Bohnert, P.R. Whitfeld, and W. Bottomley. 1982. Nucleotide sequence of the gene for the Mr 32,000 thylakoid membrane protein from Spinacia oleracea and Nicotiana debneyi predicts a totally conserved primary translation product of $\mathrm{Mr} 38,950$. Proc. Natl. Acad. Sci. 79: 7699-7703. 


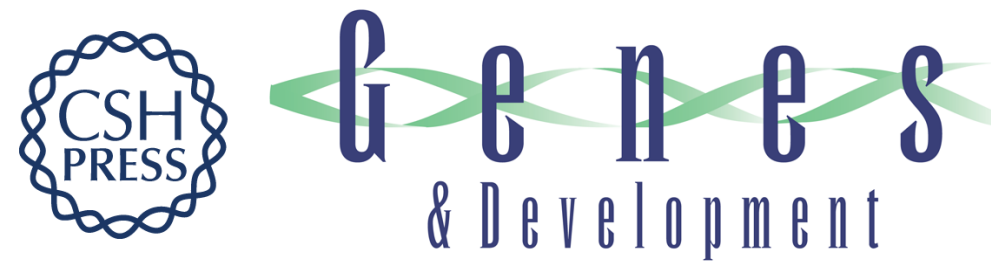

\section{cop1: a regulatory locus involved in light-controlled development and gene expression in Arabidopsis.}

X W Deng, T Caspar and P H Quail

Genes Dev. 1991, 5:

Access the most recent version at doi:10.1101/gad.5.7.1172

References This article cites 39 articles, 17 of which can be accessed free at:

http://genesdev.cshlp.org/content/5/7/1172.full.html\#ref-list-1

License

Email Alerting

Service

Receive free email alerts when new articles cite this article - sign up in the box at the top right corner of the article or click here.

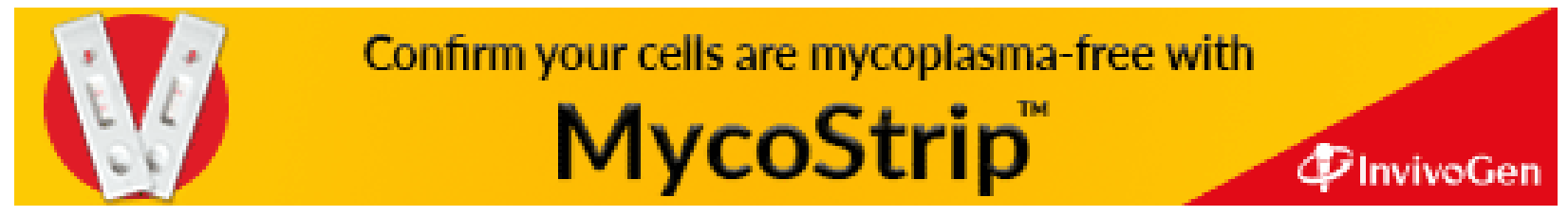

